E. GARCÍA-RÍO AND L. VANHECKE

KODAI MATH. J.

21 (1998), 46-60

\title{
HOLOMORPHIC GEODESIC TRANSFORMATIONS
}

\author{
EduARDo García-Río ${ }^{1}$ aNd LIEVEN VANHECKE
}

\begin{abstract}
We treat holomorphic geodesic transformations with respect to points and (holomorphic) submanifolds in an almost Hermitian manifold. We derive necessary and sufficient conditions for the existence and study how it influences the geometry of the submanifold. Furthermore, we use these transformations to characterize locally Hermitian symmetric spaces and complex space forms. Also, we determine all holomorphic geodesic transformations in such space forms.
\end{abstract}

\section{Introduction}

Roughly speaking, geodesic transformations with respect to points or submanifolds $P$ in a Riemannian manifold $M$ are local diffeomorphisms which transform a tubular hypersurface about $P$ into another tubular hypersurface by moving points along normal geodesics of $P$ and leaving the points of $P$ fixed. These transformations are extensions of geodesic symmetries and local reflections with respect to submanifolds. They have been introduced in [5], [13] (see also [3]). In [5]-[8], we studied conformal and divergence-preserving geodesic transformations and used them to characterize real, complex and quaternionic space forms and harmonic spaces as well as special classes of submanifolds. Isoparametric hypersurfaces in real space forms or Hopf hypersurfaces with constant principal curvatures in complex space forms are typical examples.

For an almost Hermitian manifold $M$, isometric, symplectic and holomorphic geodesic symmetries and reflections with respect to submanifolds have been treated in [2], [12] (see also [14]). As has been shown in [5], [7], an isometric or symplectic geodesic transformation reduces automatically to the identity map or a local reflection. For that reason, we focus here on the study of holomorphic geodesic transformations. In Section 2, we begin by collecting some useful

Mathematics Subject Classification. 53B20, 53B25, 53C25, 53C55.

Key words and phrases. Holomorphic geodesic transformations with respect to points and submanifolds in almost Hermitian manifolds, Fermi coordinates, complex space forms.

${ }^{1}$ Supported by projects DGICYT PB940633C0201 and XUGA 20702B96 (Spain)

Received July 28, 1997; revised November 18, 1997. 
material about the geometry of tubular neighborhoods of a submanifold and derive the first results. More precisely, we prove that the submanifold $P$ is necessarily holomorphic. Furthermore, we study the initial conditions in order to be able to detect the identity map and a local reflection. In Section 3, we derive the necessary and sufficient conditions for holomorphicity and focus on necessary conditions for the extrinsic geometry of $\boldsymbol{P}$, in particular when the ambient space is a Kähler manifold. Furthermore, in Section 4, we determine a characterization of manifolds which are locally isometric to a Hermitian symmetric space and of complex space forms by using holomorphic geodesic transformations with respect to points. Moreover, and when the ambient space $M$ is such a space form, we determine all the holomorphic geodesic transformations with respect to points and submanifolds.

\section{Holomorphic geodesic transformations. Preliminaries and first results}

Let $(M, g)$ be a connected Riemannian manifold and $\nabla$ its Levi Civita connection. $R$ denotes its Riemann curvature tensor taken with the sign convention $R_{X Y}=\nabla_{[X, Y]}-\left[\nabla_{X}, \nabla_{Y}\right]$ for all smooth vector fields $X, Y$. Furthermore, we put $R_{X Y Z W}=R(X, Y, Z, W)=g(R(X, Y) Z, W)$. Next, let $P$ be a topologically embedded submanifold. In what follows $(M, g, J)$ denotes an almost Hermitian manifold. For simplicity, we assume that all the considered data are analytic although at some places smoothness is sufficient to obtain the required result. It will be clear from the proofs where this weaker condition can be used.

Let $\exp _{v}$ denote the exponential map of the normal bundle $v$ of $P$. A geodesic transformation $\varphi_{P}$ with respect to $P$ is a map defined by

$$
\varphi_{P}: p=\exp _{v}(r u) \mapsto \varphi_{P}(p)=\exp _{v}(s(r) u)
$$

which leaves $P$ invariant (that is, $s(0)=0$ ). Here, $u$ is an arbitrary unit normal vector of $P$. Moreover, $r$ and $s$ are supposed to be sufficiently small such that $\varphi_{P}$ is a local diffeomorphism. In the rest of the paper we shall assume that $s$ is analytic in a neighborhood of $r=0$.

The geodesic transformation $\varphi_{P}$ on $(M, g, J)$ is said to be holomorphic if

$$
\varphi_{P_{*}} \circ J=J \circ \varphi_{P_{*}}
$$

To describe analytically the map $\varphi_{P}$ defined in (2.1) and the condition (2.2) we use Fermi coordinates. We briefly recall the definition and refer to [9], [10] for more details and further references. For a point $m$ of $P$, let $\left\{E_{1}, \ldots, E_{n}\right\}$, $n=\operatorname{dim} M$, be a local orthonormal frame field of $(M, g)$ defined along $P$ in a neighborhood of $m$. We specialize this field such that $E_{1}, \ldots, E_{q}, q=\operatorname{dim} P$, are tangent to $P$. For a system of coordinates $\left(y^{1}, \ldots, y^{q}\right)$ of $P$ in a neighborhood of $m$ such that $\left(\partial / \partial y^{i}\right)(m)=E_{i}(m), i=1, \ldots, q$, the Fermi coordinates 
$\left(x^{1}, \ldots, x^{n}\right)$ with respect to $m,\left(y^{1}, \ldots, y^{q}\right)$ and $\left\{E_{q+1}, \ldots, E_{n}\right\}$ are defined by

$$
\begin{cases}x^{l}\left(\exp _{v}\left(\sum_{q+1}^{n} t^{\alpha} E_{\alpha}\right)\right)=y^{l}, & i=1, \ldots, q, \\ x^{a}\left(\exp _{v}\left(\sum_{q+1}^{n} t^{\alpha} E_{\alpha}\right)\right)=t^{a}, & a=q+1, \ldots, n\end{cases}
$$

in a neighborhood of the zero section of $P$ in $v$, taken sufficiently small to have a diffeomorphic $\exp _{v}$ on that neighborhood. For a point $P$, these Fermi coordinates are the usual normal coordinates.

Now, put $s(r)=\rho(r) r$ where $r$ denotes the normal distance function to $P$. We have $r^{2}=\sum_{a=q+1}^{n}\left(x^{a}\right)^{2}$. Then $\varphi_{P}$ is described as follows:

$$
\varphi_{P}:\left(x^{1}, \ldots, x^{q}, x^{q+1}, \ldots, x^{n}\right) \mapsto\left(x^{1}, \ldots, x^{q}, \rho(r) x^{q+1}, \ldots, \rho(r) x^{n}\right) .
$$

Clearly, we have

$$
\left\{\begin{array}{l}
\varphi_{P_{*}} \frac{\partial}{\partial x^{i}}=\frac{\partial}{\partial x^{i}}, \quad i=1, \ldots, q \\
\varphi_{P_{*}} \frac{\partial}{\partial x^{a}}=\rho \frac{\partial}{\partial x^{a}}+r \rho^{\prime} \frac{\partial r}{\partial x^{a}} \frac{\partial}{\partial r}
\end{array}\right.
$$

Next, we specialize the frame field $\left\{E_{1}, \ldots, E_{n}\right\}$ such that $E_{n}(m)=u$ for $p=\exp _{v}(r u)$. The following proposition is an immediate consequence of $(2.3)$ and (2.2).

Proposition 2.1. The geodesic transformation $\varphi_{P}$ in $(M, g, J)$ is holomorphic if and only if with $J\left(\partial / \partial x^{\alpha}\right)=J_{\alpha}^{\beta}\left(\partial / \partial x^{\beta}\right), \alpha, \beta=1, \ldots, n$, we have

(2.4a) $J_{l}^{J}(p)=J_{l}^{J}\left(\varphi_{P}(p)\right)$,

(2.5a) $\rho(r) J_{i}^{b}(p)=J_{l}^{b}\left(\varphi_{P}(p)\right)$,

(2.6a) $s^{\prime}(r) J_{l}^{n}(p)=J_{l}^{n}\left(\varphi_{P}(p)\right)$,

(2.7a) $J_{n}^{J}(p)=s^{\prime}(r) J_{n}^{J}\left(\varphi_{P}(p)\right)$,

for $i, j \in\{1, \ldots, q\}$ and $a, b \in\{q+1, \ldots, n-1\}$. (2.4b) $J_{a}^{b}(p)=J_{a}^{b}\left(\varphi_{P}(p)\right)$

(2.5b) $J_{a}^{J}(p)=\rho(r) J_{a}^{J}\left(\varphi_{P}(p)\right)$,

(2.6b) $s^{\prime}(r) J_{a}^{n}(p)=\rho(r) J_{a}^{n}\left(\varphi_{P}(p)\right)$,

(2.7b) $\rho(r) J_{n}^{b}(p)=s^{\prime}(r) J_{n}^{b}\left(\varphi_{P}(p)\right)$

To prove some of our results, we will need power series expansions for the components $J_{\alpha}^{\beta}$ along a normal geodesic $\gamma: r \mapsto \exp _{v}(r u)$. We now recall a method to obtain these expansions. (See [9], [10], [14] for more details.) We start from the frame field $\left\{E_{1}, \ldots, E_{n}\right\}$ chosen above and consider the frame field $\left\{F_{1}, \ldots, F_{n}\right\}$ obtained from $\left\{E_{1}(m), \ldots, E_{n}(m)\right\}$ by parallel translation along the geodesic $\gamma$ through $m=\gamma(0)$. Furthermore, let $Y_{\alpha}, \alpha=1, \ldots, n-1$ denote the Jacobi vector fields along $\gamma$ satisfying the initial conditions 


$$
\begin{cases}Y_{i}(0)=E_{i}(m), \quad Y_{i}^{\prime}(0)=\left(\nabla_{\gamma^{\prime}} \frac{\partial}{\partial x^{i}}\right)(m), & i=1, \ldots, q, \\ Y_{a}(0)=0, \quad Y_{a}^{\prime}(0)=E_{a}(m), & a=q+1, \ldots, n-1\end{cases}
$$

where the prime denotes covariant differentiation along $\gamma$. Then we have

$$
Y_{i}(r)=\frac{\partial}{\partial x^{l}}(\gamma(r)), \quad Y_{a}(r)=r \frac{\partial}{\partial x^{a}}(\gamma(r))
$$

Next, put

$$
Y_{\alpha}(r)=D_{u}(r) F_{\alpha}(\gamma(r)), \quad \alpha=1, \ldots, n-1 .
$$

Then the Jacobi equation yields

$$
D_{u}^{\prime \prime}+R \circ D_{u}=0
$$

where $R(r) X=R_{\gamma^{\prime}(r) X} \gamma^{\prime}(r)$. To obtain the initial conditions for the endomorphism field $D_{u}$, we use the following Gauss and Weingarten equations for $P$ :

$$
\begin{aligned}
\nabla_{X} Y & =\tilde{\nabla}_{X} Y+T_{X} Y \\
\nabla_{X} \xi & =T(\xi) X+\nabla_{X}^{\perp} \xi
\end{aligned}
$$

where $X, Y$ are tangent to $P$ and where $\xi$ is a (local) normal vector field of $P$. $\quad \tilde{\nabla}$ denotes the Levi Civita connection of the induced metric on $P, T$ is the second fundamental form, $T(\xi)$ the shape operator with respect to $\xi$ and $\nabla^{\perp}$ the normal connection. $\quad T$ and $T(\xi)$ are related by $g(T(\xi) X, Y)=-g\left(T_{X} Y, \xi\right)$ for all $X, Y$ tangent to $P$. Using the initial conditions (2.8), we then obtain

$$
D_{u}(0)=\left(\begin{array}{cc}
I_{q} & 0 \\
0 & 0
\end{array}\right), \quad D_{u}^{\prime}(0)=\left(\begin{array}{cc}
T(u) & 0 \\
-{ }^{t} \perp(u) & I_{n-q-1}
\end{array}\right)
$$

where

$$
\begin{aligned}
& T(u)_{i j}=g\left(T(u) E_{l}, E_{j}\right)(m), \\
& \perp(u)_{i a}=g\left(\perp_{E_{i}} E_{a}, E_{n}\right)(m)
\end{aligned}
$$

and where $\perp$ is an operator defined in [10] which satisfies $\left(\perp_{X}(N)\right)(m)=$ $\left(\nabla_{X}^{\perp} N\right)(m)$.

Now, using the generalized Gauss lemma (see [9], [10]), we obtain

$$
g_{n n}(p)=1, \quad g_{\alpha n}(p)=0, \quad \alpha=1, \ldots, n-1,
$$

and moreover, from the formulas given above, we get

$$
\left\{\begin{array}{l}
g_{i j}(p)=\left({ }^{t} D_{u} D_{u}\right)_{i j}(r), \\
g_{i a}(p)=\frac{1}{r}\left({ }^{t} D_{u} D_{u}\right)_{i a}(r), \\
g_{a b}(p)=\frac{1}{r^{2}}\left({ }^{t} D_{u} D_{u}\right)_{a b}(r)
\end{array}\right.
$$


for $i, j=1, \ldots, q ; a, b=q+1, \ldots, n-1$. Note that we often identify the spaces $\left\{\gamma^{\prime}(r)\right\}^{\perp}$ along $\gamma$ by means of the parallel basis $\left\{F_{1}, \ldots, F_{n}\right\}$. Using (2.9)-(2.12) one may obtain power series expansions for the components $g_{\alpha \beta}$ of the metric tensor and hence also for the components $g^{\alpha \beta}$ of the inverse $g^{-1}$ of $g$. More precisely, we have [4], [14]

$$
\left\{\begin{aligned}
g_{i j}(p)= & g\left(E_{i}, E_{j}\right)(m)+2 r g\left(T(u) E_{i}, E_{j}\right)(m) \\
& +r^{2}\left\{-g\left(R(u) E_{l}, E_{j}\right)+g\left(T(u) E_{l}, T(u) E_{j}\right)\right. \\
& +g\left(^{t} \perp(u) E_{i}{ }^{t} \perp(u) E_{j}\right\}(m)+O\left(r^{3}\right) \\
g_{i a}(p)= & -r g\left(^{t} \perp(u) E_{l}, E_{a}\right)(m)-\frac{2}{3} r^{2} g\left(R(u) E_{l}, E_{a}\right)(m)+O\left(r^{3}\right) \\
g_{a b}(p)= & g\left(E_{a}, E_{b}\right)(m)-\frac{1}{3} r^{2} g\left(R(u) E_{a}, E_{b}\right)(m)+O\left(r^{3}\right)
\end{aligned}\right.
$$

where $p=\exp _{v}(r u)$.

For an almost Hermitian manifold $(M, g, J)$ we denote by $\Omega$ its Kähler form defined by $\Omega(X, Y)=g(X, J Y)$ for tangent vector fields $X, Y$. Then we have

$$
J_{\alpha}^{\beta}=-\Omega_{\alpha \gamma} g^{\nu \beta}
$$

and the desired power series expansions for $J_{\alpha}^{\beta}$ can be determined from the ones for $g^{\gamma \beta}$ and $\Omega_{\alpha \gamma}$. The expansions for $\Omega_{\alpha \gamma}$ may be obtained in a similar way as those of $g_{\alpha \beta}$. Note that an alternative method is given in [10]. We shall write down the needed expansions at the places where we use them explicitly.

We end this section with three results. First, we derive an important consequence of the existence of a holomorphic geodesic transformation $\varphi_{P}$ on the geometry of the submanifold $P$. It extends a result of [2] where it is proved that if a local reflection with respect to $P$ (that is, $s(r)=-r$ ) is holomorphic, then $P$ must be holomorphic.

THEOREM 2.1. Let $\varphi_{P}$ be a non-trivial holomorphic geodesic transformation with respect to $P$. Then $P$ is a holomorphic submanifold.

Proof. With the notations as above we consider the unit speed geodesic $\gamma: r \mapsto \exp _{v}(r u)$ and adapt the frame field $\left\{E_{1}, \ldots, E_{n}\right\}$ such that

$$
J \gamma^{\prime}(0)=\left(d E_{q}+c E_{q+1}\right)(m), \quad c^{2}+d^{2}=1 .
$$

Using this and (2.3) at $m=\gamma(0)$, we get at once

$$
s^{\prime}(0)\left(d E_{q}+c E_{q+1}\right)(m)=d E_{q}(m)+\rho(0) c E_{q+1}(m) .
$$

This holds if and only if $d\left(s^{\prime}(0)-1\right)=0$. In the next lemma, we shall show that $s^{\prime}(0)=1$ implies that $\varphi_{P}$ is the identity map. Hence, the hypothesis of non-triviality implies $d=0$ and this proves that $P$ is a holomorphic submanifold. 
LEMMA 2.1. The identity map is the only holomorphic geodesic transformation which satisfies the initial condition $s^{\prime}(0)=1$. we put

Proof. We consider the same notations as in Theorem 2.1. Furthermore,

$$
J_{\beta}^{\delta}\left(\exp _{v}(r u)\right)=\sum_{t \geq 0} \alpha_{t}(\beta, \delta) r^{t}
$$

First, we consider the case $c \neq 0$. Since $s^{\prime}(0)=1$, from (2.6b) and (2.14) we get

$$
\begin{aligned}
& \alpha_{0}(q+1, n)+\left\{\frac{1}{2} s^{\prime \prime}(0) \alpha_{0}(q+1, n)+\alpha_{1}(q+1, n)\right\} r+O\left(r^{2}\right) \\
& =\alpha_{0}(q+1, n)+\left\{s^{\prime \prime}(0) \alpha_{0}(q+1, n)+\alpha_{1}(q+1, n)\right\} r+O\left(r^{2}\right)
\end{aligned}
$$

and hence, we have $s^{\prime \prime}(0) \alpha_{0}(q+1, n)=0$. Furthermore, $\alpha_{0}(q+1, n)=J_{q+1}^{n}(m)=$ $-g\left(E_{q+1}, J E_{n}\right)(m)=-c \neq 0$ and hence, $s^{\prime \prime}(0)=0$. Next, put

$$
s(r)=r+\beta_{k+1} r^{k+1}+O\left(r^{k+2}\right)
$$

where $\beta_{k+1}, k \geq 2$, is the second non-zero coefficient in the expansion of $s(r)$. Using this in (2.6b), we obtain

$$
\begin{aligned}
& \sum_{t=0}^{k-1} \alpha_{t}(q+1, n) r^{t}+\left\{\beta_{k+1} \alpha_{0}(q+1, n)+\alpha_{k}(q+1, n)\right\} r^{k}+O\left(r^{k+1}\right) \\
& =\sum_{t=0}^{k-1} \alpha_{t}(q+1, n) r^{t}+\left\{(k+1) \beta_{k+1} \alpha_{0}(q+1, n)+\alpha_{k}(q+1, n)\right\} r^{k} \\
& \quad+O\left(r^{k+1}\right) .
\end{aligned}
$$

Considering the coefficients of $r^{k}$ in both members, we get

$$
k \alpha_{0}(q+1, n) \beta_{k+1}=0
$$

and so, $\beta_{k+1}=0$. The result $s(r)=r$ now follows by induction.

Secondly, put $c=0$. Then, by a similar procedure using (2.6a) for $J_{q}^{n}$, we obtain the same conclusion.

The above lemma shows that we can detect the identity map in the class of holomorphic geodesic transformations by means of the initial condition $s^{\prime}(0)=1$. Now, we show that we can also detect the reflection $s(r)=-r$ by means of the condition $s^{\prime}(0)=-1$.

LEMMA 2.2. The holomorphic geodesic transformation $\varphi_{P}$ is a local reflection if and only if $s^{\prime}(0)=-1$. 
Proof. We only have to prove the "if" part. We proceed as in the proof of Lemma 2.1 taking into account that $P$ is a holomorphic submanifold. So, we put $E_{q+1}(m)=J u=J \gamma^{\prime}(0)$. Then (2.6b) yields

$$
1+\left\{\alpha_{1}(q+1, n)-\frac{1}{2} s^{\prime \prime}(0)\right\} r+O\left(r^{2}\right)=1-\left\{\alpha_{1}(q+1, n)+s^{\prime \prime}(0)\right\} r+O\left(r^{2}\right)
$$

from which we get

$$
s^{\prime \prime}(0)=-4 \alpha_{1}(q+1, n):=-4 \alpha_{1}(J u, u) .
$$

Now, we replace $u$ by $u \cos \theta+J u \sin \theta$. Then we get from (2.15) by taking the limit for $\theta \rightarrow \pi$ :

$$
s^{\prime \prime}(0)=-4 \alpha_{1}(-J u,-u) .
$$

The formula for the power series expansion for $J_{q+1}^{n}$ yields that

$$
\alpha_{1}(-J u,-u)=-\alpha_{1}(J u, u)
$$

and hence, from $(2.16)-(2.17)$ we obtain that $s^{\prime \prime}(0)=0$. Next, put

$$
s^{\prime}(r)=-1+\beta_{k+1} r^{k+1}+O\left(r^{k+2}\right)
$$

where $\beta_{k+1}$ is the second non-zero coefficient, $k \geq 2$. Then, considering again (2.6b), we get

$$
\begin{aligned}
& \sum_{t=0}^{k-1}(-1)^{t} \alpha_{t}(q+1, n) r^{t}+\left\{(-1)^{k} \alpha_{k}(q+1, n)-\beta_{k+1} \alpha_{0}(q+1, n)\right\} r^{k}+O\left(r^{k+1}\right) \\
& \quad=\sum_{t=0}^{k-1} \alpha_{t}(q+1, n) r^{t}+\left\{\alpha_{k}(q+1, n)-(k+1) \beta_{k+1} \alpha_{0}(q+1, n)\right\} r^{k}+O\left(r^{k+1}\right)
\end{aligned}
$$

Since $\alpha_{0}(q+1, n)=-1$, this yields

$$
k \beta_{k+1}=\left\{(-1)^{k}-1\right\} \alpha_{k}(q+1, n) .
$$

So, for an even $k$, we get $\beta_{k+1}=0$. When $k$ is odd, we proceed as above and replace $u$ by $u \cos \theta+J u \sin \theta$. This gives

$$
k \beta_{k+1}=-2 \alpha_{k}(-J u,-u)
$$

and since $\alpha_{k}(-J u,-u)=-\alpha_{k}(J u, u)=-\alpha_{k}(q+1, n)$, we obtain $\beta_{k+1}=0$. Then $s(r)=-r$ follows by an induction procedure.

\section{Necessary and sufficient conditions. Extrinsic geometry and the}

\section{Kähler case}

We begin this section by determining the necessary and sufficient conditions for a geodesic transformation to be holomorphic and firstly consider the case of a reflection, that is, $s(r)=-r$. From Proposition 2.1 we get at once 
THEOREM 3.1. A local reflection $\varphi_{P}$ with respect to a (holomorphic) submanifold $P$ is holomorphic if and only if

$$
\begin{aligned}
& J_{l}^{j}\left(\exp _{v}(r u)\right)=J_{i}^{j}\left(\exp _{v}(-r u)\right), \\
& J_{a}^{b}\left(\exp _{v}(r u)\right)=J_{a}^{b}\left(\exp _{v}(-r u)\right), \\
& J_{l}^{b}\left(\exp _{v}(r u)\right)=-J_{l}^{b}\left(\exp _{v}(-r u)\right), \\
& J_{a}^{J}\left(\exp _{v}(r u)\right)=-J_{a}^{J}\left(\exp _{v}(-r u)\right)
\end{aligned}
$$

for $i, j \in\{1, \ldots, q\}$ and $a, b \in\{q+1, \ldots, n\}$.

Secondly, we consider the case of a geodesic transformation which is not a geodesic reflection. It will turn out that in this case the conditions are more restrictive. We use the conventions made at the end of Section 2 , that is, we take a frame field $\left\{E_{1}, \ldots, E_{n}\right\}$ such that $E_{n}(m)=u, E_{q+1}(m)=J u$. Then we have

THEOREM 3.2. Let $\varphi_{P}$ be a non-trivial geodesic transformation, different from a local reflection, with respect to a holomorphic submanifold $P$. Then $_{\varphi_{P}}$ is holomorphic if and only if the components $J_{\delta}^{\gamma}\left(\exp _{v}(r u)\right)$ are radial functions which are independent of $m \in P$ and moreover,

(a) the components $J_{\delta}^{\gamma}\left(\exp _{v}(r u)\right)$ are constant functions along normal geodesics except possibly $J_{q+1}^{n}\left(\exp _{v}(r u)\right)$ and $J_{n}^{q+1}\left(\exp _{v}(r u)\right)$;

(b) $\left(J_{q+1}^{n} J_{n}^{q+1}\right)\left(\exp _{v}(r u)=-1\right.$.

Proof. First, suppose that these conditions hold. Then the conditions $(2.4)-(2.7)$ in Proposition 2.1 reduce to $(2.6 \mathrm{~b})$ and $(2.7 \mathrm{~b})$ and because of the hypothesis (b) we are left with one single differential equation. This proves the sufficiency.

Next, we prove the necessity by taking into account that $s^{\prime}(0)^{2} \neq 1$. We rewrite the conditions given in Proposition 2.1 by means of power series expansions and take in all the cases $i, j \in\{1, \ldots, q\}$ and $a, b \in\{q+1, \ldots, n-1\}$. Then $(2.4 \mathrm{a})-(2.7 \mathrm{~b})$ yield, respectively:

$$
\begin{aligned}
\left(1-s^{\prime}(0)^{k}\right) \alpha_{k}(i, j)= & \sum_{l=0}^{k-1} \alpha_{l}(i, j)\left(\sum_{p_{1}+\cdots+p_{l}=k} \beta_{p_{1}} \cdots \beta_{p_{l}}\right), \\
\left(1-s^{\prime}(0)^{k}\right) \alpha_{k}(a, b)= & \sum_{l=0}^{k-1} \alpha_{l}(a, b)\left(\sum_{p_{1}+\cdots+p_{l}=k} \beta_{p_{1}} \cdots \beta_{p_{l}}\right) \\
s^{\prime}(0)\left(1-s^{\prime}(0)^{k-1}\right) \alpha_{k}(i, b)= & -\sum_{t+l=k+1} \beta_{t} \alpha_{l}(i, b) \\
& +\sum_{l=0}^{k-1} \alpha_{l}(i, b)\left(\sum_{p_{1}+\cdots+p_{l}=k} \beta_{p_{1}} \cdots \beta_{p_{l}}\right)
\end{aligned}
$$




$$
\begin{aligned}
\left(1-s^{\prime}(0)^{k+1}\right) \alpha_{k}(a, j)= & \beta_{1} \sum_{l=0}^{k-1} \alpha_{l}(a, j)\left(\sum_{p_{1}+\cdots+p_{l}=k} \beta_{p_{1}} \cdots \beta_{p_{l}}\right) \\
& +\sum_{\substack{t+v=k+1 \\
t>1}} \beta_{t}\left(\sum_{l \geq 0} \alpha_{l}(a, j) \sum_{p_{1}+\cdots+p_{l}=v} \beta_{p_{1}} \cdots \beta_{p_{l}}\right) \\
s^{\prime}(0)\left(1-s^{\prime}(0)^{k-1}\right) \alpha_{k}(i, n)= & -\sum_{\substack{t+l=k+1 \\
t>1}} t \beta_{t} \alpha_{l}(i, n) \\
& +\sum_{l=0}^{k-1} \alpha_{l}(i, n)\left(\sum_{p_{1}+\cdots+p_{l}=k} \beta_{p_{1}} \cdots \beta_{p_{l}}\right)
\end{aligned}
$$

$$
\begin{aligned}
s^{\prime}(0)\left(1-s^{\prime}(0)^{k}\right) \alpha_{k}(a, n)= & -\sum_{\substack{t+l=k+1 \\
t>1}} t \beta_{t} \alpha_{l}(a, n) \\
& +\beta_{1} \sum_{l=0}^{k-1} \alpha_{l}(a, n)\left(\sum_{p_{1}+\cdots+p_{l}=k} \beta_{p_{1}} \cdots \beta_{p_{l}}\right) \\
& +\sum_{\substack{t+v=k+1 \\
t>1}} \beta_{t}\left(\sum_{l \geq 0} \alpha_{l}(a, n) \sum_{p_{1}+\cdots+p_{l}=v} \beta_{p_{1}} \cdots \beta_{p_{l}}\right)
\end{aligned}
$$

$$
\begin{aligned}
\left(1-s^{\prime}(0)^{k+1}\right) \alpha_{k}(n, j)= & \beta_{1} \sum_{l=0}^{k-1} \alpha_{l}(n, j)\left(\sum_{p_{1}+\cdots+p_{l}=k} \beta_{p_{1}} \cdots \beta_{p_{l}}\right) \\
& +\sum_{\substack{t+v=k+1 \\
t>1}} t \beta_{t}\left(\sum_{l \geq 0} \alpha_{l}(n, j) \sum_{p_{1}+\cdots+p_{l}=v} \beta_{p_{1}} \cdots \beta_{p_{l}}\right)
\end{aligned}
$$

$$
\begin{aligned}
s^{\prime}(0)\left(1-s^{\prime}(0)^{k}\right) \alpha_{k}(n, b)= & -\sum_{\substack{t+l=k+1 \\
t>1}} \beta_{t} \alpha_{l}(n, b) \\
& +\beta_{1} \sum_{l=0}^{k-1} \alpha_{l}(n, b)\left(\sum_{p_{1}+\cdots+p_{l}=k} \beta_{p_{1}} \cdots \beta_{p_{l}}\right) \\
& +\sum_{\substack{t+v=k+1 \\
l>1}} t \beta_{t}\left(\sum_{l \geq 0} \alpha_{l}(n, b) \sum_{p_{1}+\cdots+p_{l}=v} \beta_{p_{1}} \cdots \beta_{p_{l}}\right)
\end{aligned}
$$

where $J_{\delta}^{\gamma}\left(\exp _{y}(r u)\right)=\sum_{k \geq 0} \alpha_{k}(\delta, \gamma) r^{k}$ and $s(r)=\sum_{l \geq 1} \beta_{l} r^{l}$.

Now, using (3.3)-(3.10), an induction procedure yields that the $\alpha_{k}(\delta, \gamma)$ are independent of $m$ and $u$. Moreover, the same formulas show that each co- 
efficient $\alpha_{k}$ is completely determined by the coefficients $\alpha_{l}$ for $l<k$ and by the coefficients $\beta_{t}$. Hence, if $\alpha_{0}$ vanishes, then $J_{\delta}^{\gamma}\left(\exp _{v}(r u)\right)=0$. Moreover, for $\delta, \gamma \in\{1, \ldots, q\}$ or $\{q+1, \ldots, n-1\},(3.3)$ and (3.4) yield $\alpha_{1}=0$ and then, using again an induction procedure, we obtain $\alpha_{t}=0$ for all $t \geq 1$. This shows that $J_{q+1}^{n}\left(\exp _{v}(r u)\right)$ and $J_{n}^{q+1}\left(\exp _{v}(r u)\right)$ are constant along normal geodesics. This proves statement $(\mathrm{a})$.

Finally, $\varphi_{P}$ must satisfy $(2.6 \mathrm{~b})$ and $(2.7 \mathrm{~b})$. From this, we get

$$
F(r)=F(s)
$$

for $F(r)=\left(J_{q+1}^{n} J_{n}^{q+1}\right)\left(\exp _{v}(r u)\right)$. Since $F(0)=-1$ and proceeding as before, we get $F(r)=-1$. This proves statement (b).

Next, we focus on some consequences concerning the extrinsic geometry of the holomorphic submanifold $P$. In what follows we only consider non-trivial geodesic transformations without mentioning it explicitly.

THEOREM 3.3. Let $\varphi_{P}$ be a holomorphic geodesic transformation with respect to $P$. Then we have

$$
T(J X, J Y)-T(X, Y)=0
$$

for $X, Y \in T P$ and moreover, $\left(\nabla_{u} J\right) X$ is normal and $\left(\nabla_{u} J\right) V$ tangent to $P$ for all $u$, $V \in T^{\perp} P$ and $X \in T P$.

Proof. Using the method described in Section 2, we get

$$
\begin{aligned}
-J_{l}^{J}\left(\exp _{v}(r u)\right)= & g\left(E_{l}, J E_{J}\right)(m)+r\left\{g\left(E_{l},\left(\nabla_{u} J\right) E_{J}\right)+g\left(T(u) E_{l}, J E_{J}\right)\right. \\
& \left.+g\left(E_{l}, J T(u) E_{J}\right)+2 g\left(T(u) J E_{i}, E_{J}\right)\right\}(m)+O\left(r^{2}\right) .
\end{aligned}
$$

Proceeding as in [2, Theorem 10], we get (3.11).

Moreover, since $g\left(E_{l},\left(\nabla_{u} J\right) E_{j}\right)$ must vanish for all $i, j \in\{1, \ldots, q\}$, we get that $\left(\nabla_{u} J\right) X$ must be normal to $P$ for all $X \in T P$. Finally, since

$$
-J_{a}^{b}\left(\exp _{v}(r u)\right)=g\left(E_{a}, J E_{b}\right)(m)+r g\left(E_{a},\left(\nabla_{u} J\right) E_{b}\right)(m)+O\left(r^{2}\right),
$$

we have $g\left(E_{a},\left(\nabla_{u} J\right) E_{b}\right)=0$ which shows that $\left(\nabla_{u} J\right) V$ is tangent to $P$ for all $V \in T^{\perp} P$.

Furthermore, concerning the normal component of $\left(\nabla_{u} J\right) X, X \in T P$, we have

THEOREM 3.4. Let $\varphi_{P}$ be a holomorphic geodesic transformation which is not a local reflection. Then we have

$$
\left(\nabla_{u} J\right) X=\left(J^{t} \perp(u)-{ }^{t} \perp(u) J\right) X
$$

for all $X \in T P$ and $u \in T^{\perp} P$. 
Proof. Using again the method described in Section 2, we get

$$
\begin{aligned}
\Omega_{i k}\left(\exp _{v}(r u)\right)= & g\left(E_{l}, J E_{k}\right)(m)+r\left\{g\left(\left(\nabla_{u} J\right) E_{k}, E_{l}\right)+g\left(T(u) E_{i}, J E_{k}\right)\right. \\
& \left.-g\left(T(u) E_{k}, J E_{l}\right)\right\}(m)+O\left(r^{2}\right), \\
\Omega_{i c}\left(\exp _{v}(r u)\right)= & r\left\{g\left(\left(\nabla_{u} J\right) E_{c}, E_{l}\right)-g\left({ }^{t} \perp(u) E_{i}, J E_{c}\right)\right\}(m)+O\left(r^{2}\right)
\end{aligned}
$$

for $i, j \in\{1, \ldots, q\}$ and $c \in\{q+1, \ldots, n\}$. Hence, we obtain

$$
\begin{aligned}
-J_{i}^{b}\left(\exp _{v}(r u)\right)= & r\left\{g\left(E_{b}, J^{t} \perp(u) E_{l}\right)-g\left(E_{b},{ }^{t} \perp(u) J E_{i}\right)\right. \\
& \left.-g\left(E_{b},\left(\nabla_{u} J\right) E_{l}\right)\right\}(m)+O\left(r^{2}\right) .
\end{aligned}
$$

So, this and Theorem 3.2 yield the required formula (3.12) since $\left(\nabla_{u} J\right) E_{l}$ is normal to $P$ (Theorem 3.3).

Now, we restrict to Kähler geometry and derive some consequences from the above results.

THEOREM 3.5. Let $(M, g, J)$ be a Kähler manifold and $P$ a holomorphic submanifold such that $\varphi_{P}$ is a non-isometric holomorphic geodesic transformation. Then $P$ is totally geodesic and the normal connection satisfies $\nabla_{J X}^{\perp} J Y+$ $\nabla_{X}^{\perp} Y=0$ for all $X \in T P$ and $Y \in T^{\perp} P$.

Proof. For a Kähler manifold we have, since $P$ is holomorphic,

$$
T(X, Y)+T(J X, J Y)=0
$$

and hence, from this and (3.11), we obtain $T=0$, that is, $P$ is totally geodesic. Moreover, since $\varphi_{P}$ is non-isometric, it is neither the identity map nor a local reflection (see Remark 4.1). Hence, from (3.12) we get

$$
\perp(u) \circ J=J \circ \perp(u) \text {. }
$$

Using the definition of $\perp$, this implies

$$
\nabla_{J X}^{\perp} Y=J \nabla_{X}^{\perp} Y
$$

for all $X \in T P$ and $Y \in T^{\perp} P$. From this the result follows at once.

THEOREM 3.6. Let $(M, g, J)$ be a Kähler manifold and $\varphi_{P}$ a holomorphic geodesic transformation with respect to $P$. Then $R(u, J u) u$ is normal to $P$ for all $u \in T^{\perp} P$. Moreover, if $\varphi_{P}$ is not a local reflection, then $R(u, J u) u$ is proportional to $J u$.

Proof. Since $(M, g, J)$ is Kählerian, it follows from the proof of Theorem 3.5 that $P$ is totally geodesic. Then we get

$$
-J_{l}^{n}\left(\exp _{v}(r u)\right)=r g\left({ }^{t} \perp(u) E_{l}, J E_{n}\right)(m)-\frac{1}{2} r^{2} R\left(u, J u, u, E_{\imath}\right)(m)+O\left(r^{3}\right) .
$$


Now, it follows from Theorem 3.1 and Theorem 3.2 that $R\left(u, J u, u, E_{\imath}\right)$ must vanish and hence $R(u, J u) u$ is normal to $P$. Furthermore, we have

$$
-J_{a}^{n}\left(\exp _{v}(r u)\right)=g\left(E_{a}, J E_{n}\right)(m)-\frac{1}{6} r^{2} R\left(u, J u, u, E_{a}\right)(m)+O\left(r^{3}\right) .
$$

Now, if $\varphi_{P}$ is not a local reflection, then Theorem 3.2 yields that $J_{a}^{n}$ is constant along a normal geodesic for $a \in\{q+2, \ldots, n-1\}$ where $E_{q+1}(m)=J u$. So, $R\left(u, J u, u, E_{a}\right)=0$ and this implies the result.

\section{Holomorphic geodesic transformations and complex space forms}

We begin with a characterization of manifolds which are locally isometric to a Hermitian symmetric space and of complex space forms.

TheOREM 4.1. Let $(M, g, J)$ be an almost Hermitian manifold. Then it is locally isometric to a Hermitian symmetric space if and only if there exists a holomorphic geodesic transformation $\varphi_{m}$ with respect to each point $m$ of $M$. Furthermore, it is a complex space form if there also exists a holomorphic geodesic transformation, which is not a local reflection, for some point $m \in M$.

Proof. When $P$ reduces to a point $m$ of $M$, the Fermi coordinates are the usual normal coordinates and then we have

$$
\begin{aligned}
-J_{a}^{b}\left(\exp _{m}(r u)\right)= & \left(g^{c b} \Omega_{a c}\right)(m)+r\left(g^{c b} \nabla_{u} \Omega_{a c}\right)(m) \\
& +r^{2}\left\{\frac{1}{2} g^{c b} \nabla_{u u}^{2} \Omega_{a c}-\frac{1}{6} g^{c b}\left(R_{u a u J c}-R_{u c u J a}\right)+\frac{1}{3} R_{u c u b} \Omega_{a c}\right\}(m) \\
& +r^{3}\left\{\frac{1}{6} g^{c b} \nabla_{u u u}^{3} \Omega_{a c}-\frac{1}{6} g^{c b} \sum_{t}\left(R_{u a u t} \nabla_{u} \Omega_{t c}+R_{u t u c} \nabla_{u} \Omega_{a t}\right)\right. \\
& -\frac{1}{12} g^{c b}\left(\nabla_{u} R_{u a u J c}-\nabla_{u} R_{u c u J a}\right)+\frac{1}{6} \sum_{c} \nabla_{u} R_{u c u b} \Omega_{a c} \\
& \left.+\frac{1}{3} \sum_{c} R_{u c u b} \nabla_{u} \Omega_{a c}\right\}(m)+O\left(r^{4}\right) .
\end{aligned}
$$

Now, we use Theorem 3.1 and Theorem 3.2. It follows that the coefficient of $r$ must vanish and hence $\nabla J=0$. So, $(M, g, J)$ is a Kähler manifold. Furthermore, the vanishing of the coefficient of $r^{3}$ then yields

$$
\nabla_{u} R_{u a u J b}-\nabla_{u} R_{u b u J a}+2 \nabla_{u} R_{u J a u b}=0 .
$$

By substituting $E_{a}(m)$ by $u$ and $E_{b}(m)$ by $J u$ in this relation, we get $\nabla_{u} R_{u J u u J u}=0$ and this implies that $(M, g, J)$ is locally isometric to a Hermitian symmetric space (see, for example, [12]).

The converse is well-known. 
Finally, suppose now that for some point $m \in M, \varphi_{m}$ is not a local reflection. Then it follows from Theorem 3.6 and [11] that $(M, g, J)$ is a complex space form.

The characterization of complex space forms follows from this theorem and the following classification theorem.

THEOREM 4.2. Let $M(c)$ be a complex space form of constant holomorphic sectional curvature $c$. Then we have the following holomorphic geodesic transformations:

(1) $\varphi_{m}$ is a local reflection (or equivalently, $\varphi_{m}$ is an isometry);

(2) $\varphi_{m}$ is given by $s(r)=C r, C \in R$ and $C^{2} \neq 0,1$, if $c=0$;

(3) $\varphi_{m}$ is determined by

$$
\tan s \frac{\sqrt{c}}{2}=C \tan r \frac{\sqrt{c}}{2}, \quad C \in R, \quad C^{2} \neq 0,1
$$

if $c>0$

(4) $\varphi_{m}$ is determined by

if $c<0$.

$$
\tanh s \frac{\sqrt{-c}}{2}=C \tanh r \frac{\sqrt{-c}}{2}, \quad C \in R, \quad C^{2} \neq 0,1
$$

Proof. First, for a complex space form, every local reflection with respect to a point is isometric and holomorphic.

Next, we determine the non-isometric $\varphi_{m}$ for an $M(c)$. We consider the case where $c$ is positive. The two other cases are similar. Using the technique given in Section 2, a straightforward computation yields

$$
\left\{\begin{array}{l}
\frac{\partial}{\partial x^{1}}(\gamma(r))=\left(\frac{1}{r \sqrt{c}} \sin r \sqrt{c}\right) J F_{n}(\gamma(r)), \\
\frac{\partial}{\partial x^{a}}(\gamma(r))=\left(\frac{2}{r \sqrt{c}} \sin r \sqrt{c}\right) F_{a}(\gamma(r))
\end{array}\right.
$$

for $F_{1}=J F_{n}$ and $a \in\{2, \ldots, n-1\}$. From this we get

$$
\begin{aligned}
& J_{1}^{a}=0, \quad J_{a}^{1}=0, \quad J_{n}^{a}=0, \quad J_{a}^{n}=0, \\
& J_{1}^{n}(\gamma(r))=\frac{-1}{r \sqrt{c}} \sin r \sqrt{c}, \\
& J_{n}^{1}(\gamma(r))=\left(\frac{1}{r \sqrt{c}} \sin r \sqrt{c}\right)^{-1}, \\
& J_{a}^{b}(\gamma(r))=g\left(J F_{a}, F_{b}\right)(\gamma(r))=g\left(J E_{a}, E_{b}\right) .
\end{aligned}
$$

Hence, the conditions given in Theorem 3.2 are satisfied. Moreover, the defining differential equation (2.6b) (or equivalently, (2.7b)) 
is

$$
r s^{\prime}(r) J_{1}^{n}(\gamma(r))=s(r) J_{1}^{n}(\gamma(s))
$$

Hence, we have

$$
\frac{d r}{\sin r \sqrt{c}}=\frac{d s}{\sin s \sqrt{c}}
$$

$$
\tan s \frac{\sqrt{c}}{2}=C \tan r \frac{\sqrt{c}}{2} .
$$

Remark 4.1. A.- It has been proved in [4] that a local reflection is harmonic if and only if it is an isometry. Furthermore, as is well-known, any holomorphic map in a Kähler manifold is harmonic (see [4] for references). Hence, it follows that the transformations given in (2), (3) and (4) of Theorem 4.2 are non-isometric harmonic maps.

B.- Note that the defining relations in (2), (3) and (4) also define conformal geodesic transformations in real space forms [5].

We finish this section with the consideration of holomorphic geodesic transformations with respect to submanifolds $P$ of dimension $\geq 1$ in complex space forms. Then the situation is quite different. We have

THEOREM 4.3. Let $M(c)$ be a complex space form and $\varphi_{P}$ a non-trivial holomorphic geodesic transformation with respect to a holomorphic submanifold $P$. Then we have

(i) $\varphi_{P}$ is isometric and hence a local reflection with respect to a totally geodesic $P$, or

(ii) $M(c)$ is locally flat, $P$ totally geodesic and $\varphi_{P}$ is determined by $s(r)=C r$, $C^{2} \neq 0,1$

and conversely.

Proof. If $\varphi_{P}$ is an isometry, then $P$ is totally geodesic and moreover, $\varphi_{P}$ is a local reflection [8]. Conversely, if $\varphi_{P}$ is a local reflection with respect to a holomorphic totally geodesic $P$, then $\varphi_{P}$ is an isometry [2, Corollary 4] and it is also holomorphic [2, Corollary 20].

Next, let $\varphi_{P}$ be non-isometric. Then Theorem 3.5 implies that $P$ is totally geodesic. Using the Ricci equation (see [1]) and the condition for the normal connection given in Theorem 3.5, it then follows that $R^{\perp}(X, J X, Y, J Y)=$ $(c / 2) g(X, X) g(Y, Y)=0$ for $X \in T P, Y \in T^{\perp} P$ and hence, $c=0$.

Conversely, if $(M, g)$ is locally flat, we get with the usual choice of Fermi coordinates as before:

$$
\frac{\partial}{\partial x^{l}}(\gamma(r))=F_{i}(\gamma(r)), \quad \frac{\partial}{\partial x^{q+1}}(\gamma(r))=J F_{n}(\gamma(r)), \quad \frac{\partial}{\partial x^{a}}(\gamma(r))=F_{a}(\gamma(r))
$$

where $i \in\{1, \ldots, q\}$ and $a \in\{q+1, \ldots, n-1\}$. Then the result follows from Theorem 3.2 by proceeding as in the proof of Theorem 4.2.

Remark 4.2. For Kähler manifolds which are not complex space forms, the situation is different. For example, an explicit calculation as in Theorem 4.2 or 
Theorem 4.3 shows that the relations given in Theorem 4.2 (2), (3), (4) determine holomorphic geodesic transformations with respect to an almost Hermitian manifold $\boldsymbol{P}$ where $\boldsymbol{P}$ is considered as a totally geodesic holomorphic submanifold in the product $P \times M(c)$.

\section{REFERENCES}

[1] B. Y. ChEN, Geometry of Submanifolds, Pure Appl. Math., 22, Marcel Dekker, New York, 1973.

[2] B. Y. Chen AND L. VANHECKE, Isometric, holomorphic and symplectic reflections, Geom. Dedicata, 29 (1989), 259-277.

[ 3 ] J. E. D'ATrI, Geodesic conformal transformations and symmetric spaces, Kōdai Math. Sem. Rep., 26 (1975), 201-203.

[4] S. Donnin, G. Gigante AND L. VANHECKE, Harmonic reflections with respect to submanifolds, Illinois J. Math., 34 (1990), 78-86.

[5] E. García-Río AND L. VANHECKE, Geodesic transformations and space forms, Math. J. Toyama Univ., 20 (1997), 57-77.

[6] E. García-Río AND L. VANHECKE, Geodesic transformations and harmonic spaces, to appear in Rend. Circ. Mat. Palermo.

[7] E. GaRcíA-Río AND L. VANHECKE, Divergence-preserving geodesic transformations, to appear in Proc. Roy. Soc. Edinburgh

[8] E. García-Río AND L. VANHECKE, Conformal geodesic transformations, to appear in Arab J. Math. Sci.

[9] A. Gray, Tubes, Addition-Wesley Publ. Co., Redwood, 1990.

[10] A. Gray and L. VANHECKE, The volumes of tubes in a Riemannian manifold, Rend. Sem. Mat. Univ. Politec. Torino, 39 (1981), 1-50.

[11] K. Nomzu, Conditions for the constancy of the holomorphic sectional curvature, J. Differential Geom., 8 (1973), 335-339.

[12] K. SekigaWA AND L. VanheCKE, Symplectic geodesic symmetries on Kähler manifolds, Quart. J. Math. Oxford, 37 (1986), 95-103.

[13] S. TaChibana, On Riemannian spaces admitting geodesic conformal transformations, Tensor N. S., 25 (1972), 323-331.

[14] L. VANHECKE, Geometry in normal and tubular neighborhoods, Rend. Sem. Fac. Sci. Univ. Cagliari, 58 (1988), suppl., 73-176.

Facultade de Matemáticas

Universidade de SANTIAGo de Compostela

15706 Santiago de Compostela, Spain

e-mail: eduardo@zmat.usc.es

Department of Mathematics

KATHOLIEKE UNIVERSTTEIT LEUVEN

Celestungnlaan 200B, 3001 Leuven, Belgium

e-mail: lieven.vanhecke@wis.kuleuven.ac.be 